\title{
ON THE EXISTENCE OF FIELDS IN BOOLEAN ALGEBRAS*
}

\author{
BY \\ B. A. BERNSTEIN
}

A class $K$ is said to be a field with respect to a pair of operations $\Delta$, $O$ if $\Delta, O$ play in $K$ the same rôle which the operations,$+ \times$ play in the class of rational numbers. My aim in this paper is to determine in any boolean algebra all pairs of operations expressible in terms of addition, multiplication, and negation for which the elements are a field.

Postulates for fields. The conditions that make a class $K$ a field with respect to a pair of operations $\Delta$, $O$ are $\dagger$ given by the following seven postulates :

P. $x \Delta y=y \Delta x$, if $x, y, y \Delta x$ are $K$-elements.

$\mathrm{P}_{2} . \quad(x \Delta y) \Delta z=x \Delta(y \Delta z)$, if $x, y, z, x \Delta y, y \Delta z, x \Delta(y \Delta z)$ are $K$-elements.

$\mathrm{P}_{3}$. For any two $K$-elements $x, y$ there is a $K$-element $\xi$ such that $x \Delta \xi=y$.

$\mathrm{P}_{4}$. $x \bigcirc y=y \bigcirc x$, if $x, y, y \bigcirc x$ are $K$-elements.

$\mathrm{P}_{5}$. $\quad(x \circ y) \bigcirc z=x \bigcirc(y \bigcirc z)$, if $x, y, z, x \bigcirc y, y \bigcirc z, x \bigcirc(y \bigcirc z)$ are $K$-elements.

P. $x \bigcirc(y \Delta z)=(x \circ y) \Delta(x \bigcirc z)$, if $x, y, z, y \Delta z, x \bigcirc y, x \bigcirc z,(x \circ y) \Delta(x \bigcirc z)$ are $K$-elements.

$P_{7}$. For any two $K$-elements $x, y$ satisfying the conditions $x \Delta x \neq x, y \Delta y \neq y$, there is a $K$-element $\eta$ such that $x O \eta=y$.

Conditions on boolean operations imposed by the postulates. In a boolean algebra any binary operation expressible in terms of addition, multiplication, and negation must be of the form

$$
A x y+B x y^{\prime}+C x^{\prime} y+D x^{\prime} y^{\prime}, \S
$$

where $A, B, C, D$ are elements of the algebra. Let two boolean operations $\Delta, O$ be given by

* Presented to the Society, San Francisco Section, April 4, 1925; received by the editors in December, 1925.

$\dagger$ Of a pair of field operations, the operation appearing first will be the one possessing the properties that " + " has in the algebra of rationals.

$\ddagger$ See E. V. Huntington, these Transactions, vol. 4 (1903), p. 31.

$\S$ Schröder's notation will be employed throughout, except that $x^{\prime}$ (instead of $x_{1}$ ) will be used for the negative of $x$. 


$$
\begin{aligned}
& x \Delta y=a x y+b x y^{\prime}+c x^{\prime} y+d x^{\prime} y^{\prime}, \\
& x \circ y=p x y+q x y^{\prime}+r x^{\prime} y+s x^{\prime} y^{\prime} .
\end{aligned}
$$

I proceed to find the conditions imposed by the postulates $P_{1}-P_{7}$ on the coefficients of (1) and(2).

The conditions that (1), (2) satisfy $P_{1}, P_{2}, P_{3}, P_{4}, P_{6}, P_{6}$ are* respectively

$$
\begin{array}{lc}
\text { (3) } & c=b, \\
\text { (4) } & a^{\prime} d+\left(a d+a^{\prime} d^{\prime}\right)\left(b c^{\prime}+b^{\prime} c\right)=0, \\
\text { (5) } & b=a^{\prime}, d=c^{\prime}, \\
(6) & r=q, \\
\text { (7) } & p^{\prime} s+\left(p s+p^{\prime} s^{\prime}\right)\left(q r^{\prime}+q^{\prime} r\right)=0, \\
\text { (8) } & a^{\prime}(p q+r s)+d\left(p^{\prime} q^{\prime}+r^{\prime} s^{\prime}\right)+\left(a d+a^{\prime} d^{\prime}+b c+b^{\prime} c^{\prime}\right)\left(p^{\prime} q+r^{\prime} s\right)=0 .
\end{array}
$$

To find the condition that (1), (2) satisfy $\mathrm{P}_{7}$, note first that the condition $x \Delta x \neq x$ is the same as $a^{\prime} x+d x^{\prime} \neq 0$. Further, the condition that for given $x, y$ there be an element $\eta$ such that $x \bigcirc \eta=y$, is the condition of solvability in $\eta$ of the equation

i. e. of the equation

$$
p x \eta+q x \eta^{\prime}+r x^{\prime} \eta+s x^{\prime} \eta^{\prime}=y,
$$

$\left(p^{\prime} x y+p x y^{\prime}+r^{\prime} x^{\prime} y^{\prime}+r x^{\prime} y^{\prime}\right) \eta+\left(q^{\prime} x y+q x y^{\prime}+s^{\prime} x^{\prime} y+s x^{\prime} y^{\prime}\right) \eta^{\prime}=0$. The condition for the solvability of this equation is

$$
p^{\prime} q^{\prime} x y+p q x y^{\prime}+r^{\prime} s^{\prime} x^{\prime} y+r s x^{\prime} y^{\prime}=0 .
$$

Hence the condition that (1), (2) satisfy $\mathrm{P}_{7}$ is

(9) $p^{\prime} q^{\prime} x y+p q x y^{\prime}+r^{\prime} s^{\prime} x^{\prime} y+r s x^{\prime} y^{\prime}=0, a^{\prime} x+d x^{\prime} \neq 0, a^{\prime} y+d y^{\prime} \neq 0$.

By (3)-(5), the condition that (1) satisfy $\mathrm{P}_{1}-\mathrm{P}_{3}$ simultaneously is

$$
b=c=d^{\prime}=a^{\prime} . \dagger
$$

By (6), (7), the condition that (2) satisfy $P_{4}, P_{5}$ simultaneously is

$$
r=q, p^{\prime} s=0 .
$$

By (8), (10), (11), the conditions that (1), (2) satisfy $P_{1}-P_{6}$ simultaneously are

* For (4), (5) see my Operations with respect to which the elements of a boolean algebra form a group, these Transactions, vol. 26 (1924), pp. 171-175, and E. Schröder, Vorlesungen über die Algebra der Logik, vol. 2, part 2, pp. 493, 494. My results were obtained without the knowledge of Schröder's work. Condition (8) I take from Schröder.

† So that all operations (1) with respect to which the elements of a boolean algebra form an abelian group, are given by $a x y+a^{\prime} x y^{\prime}+a^{\prime} x^{\prime} y+a x^{\prime} y^{\prime}$. Compare my paper cited above. 


$$
b=c=d^{\prime}=a^{\prime}, r=q, a^{\prime} q+p^{\prime} q+q^{\prime} s+a p^{\prime} q^{\prime}+a q^{\prime} s^{\prime}=0,
$$

which reduce to

$$
b=c=d^{\prime}=a^{\prime}, r=q=a, a p^{\prime}+a^{\prime} s=0 .
$$

By (10), if (1) satisfy $\mathrm{P}_{1}-\mathrm{P}_{3}$ then $d=a$, and the condition $a^{\prime} x+d x^{\prime} \neq 0$ in (9) becomes $a^{\prime} x+a x^{\prime} \neq 0$, i. e. $x \neq a$. It is to be observed that (9), (12) are sufficient as well as necessary conditions that (1), (2) satisfy $\mathrm{P}_{\mathbf{1}}-\mathrm{P}_{7_{\mathbf{7}}}$. Hence

THEOREM 1. The necessary and sufficient conditions that (1), (2) satisfy $\mathrm{P}_{1}-\mathrm{P}_{7}$ simultaneously are

$$
\begin{gathered}
x \Delta y=a x y+a^{\prime} x y^{\prime}+a^{\prime} x^{\prime} y+a x^{\prime} y^{\prime}, \\
x \circ y=p x y+a x y^{\prime}+a x^{\prime} y+s x^{\prime} y^{\prime}, \\
a p^{\prime}+a^{\prime} s=0, \\
a^{\prime} p^{\prime} x y+a p x y^{\prime}+a^{\prime} s^{\prime} x^{\prime} y+a s x^{\prime} y^{\prime}=0, x \neq a, y \neq a .
\end{gathered}
$$

Existence of fields. Conditions (13)-(16) do not, of course, prove that fields in boolean algebras exist. To consider existence, take first the algebra consisting of the boolean elements 0,1 . Let $a=0$ in (13)-(16). Then from (15), $s=0$. Letting $x=1, y=1$ in (16), we get $p=1$. Hence, the boolean elements 0,1 are a field for the pair of operations

$$
x y^{\prime}+x^{\prime} y, x y \text {. }
$$

Let $a=1$ in (13)-(16). Then from (15), $p=1$. From (16), by letting $x=0$, $y=0$, we get $s=0$. So that the boolean elements 0,1 are a field for the pair of operations

$$
x y+x^{\prime} y^{\prime}, x y+x y^{\prime}+x^{\prime} y=x+y .
$$

Since in a two-element boolean algebra all binary operations satisfying the condition of closure are of the form (1), ${ }^{*}$ we observe from (13) that operations (17), (18) are the only pair of operations for which the boolean elements 0,1 are a field.

Turning to a boolean algebra of more than two elements, let $e$ be an element different from 0,1 . The element $a$ in (13)-(16) must be 0,1 , or an element different from 0, 1. If $a=0$, then $s=0$. But by letting $x=e^{\prime}, y=e$ in (16), we get $e=0$, contrary to hypothesis. We obtain a similar contradiction for $a=1$. Let $a$ be different from 0,1. From (16), making the substitutions

$$
x=1, y=1 ; x=1, y=0 ; x=0, y=1 ; x=0, y=0,
$$

- See my Complete sets of representations of two-element algebras, Bulletin of the American Mathematical Society, vol. 30 (1924), p. 26. This paper also contains a different proof of the fact that (17), (18) are field operations for $0,1$. 
we get respectively

Hence

$$
a^{\prime} p^{\prime}=0, a p=0, a^{\prime} s^{\prime}=0, a s=0 .
$$

$$
s=p=a^{\prime} .
$$

But this contradicts (15). We therefore find that in a boolean algebra having more than two elements there exist no operations (1), (2) for which the elements are a field.

To sum up, we have

THEOREM 2. In a two-element boolean algebra there exist two and only two pairs of operations for which the elements are a field, namely

$$
x y^{\prime}+x^{\prime} y, x y ; x y+x^{\prime} y^{\prime}, x+y \text {. }
$$

In a boolean algebra of more than two elements there exist no operations expressible in terms of addition, multiplication, and negation for which the elements are a field.

UnIversity of California, Berkeley, Calif. 